\title{
Avaliação de curvas de crescimento em suínos
}

\author{
Nascimento, C.A.M.S. ${ }^{\text {; }}$ Ribeiro, M.N. ${ }^{2}$; Rocha, L.L. ${ }^{3}$ e Lucena, L.R.R. ${ }^{3}$
}

'BEXT-UAST/UFRPE. Brasil.

${ }^{2}$ Departamento de Zootecnia. UFRPE. Recife. Pernambuco. Brasil.

${ }^{3}$ Unidade Acadêmica de Serra Talhada. UFRPE. Serra Talhada. Pernambuco. Brasil.

\section{PALAVRAS-CHAVE ADICIONAIS}

Modelos de regressão.

Funç̃o não linear.

Idade.

Monogástrico.

\section{RESUMO}

Objetivou-se simular pesos de suínos machos e fêmeas (de 1 a 154 dias de idade) através da distribuição de probabilidade uniforme num intervalo [a,b]. Foram ajustados os modelos de regressão (logístico, potência, tangente hiperbólica, gamma e polinomial cúbico) para explicar a possível relação do peso com o número de dias dos suínos. Verificou-se que os modelos logístico, gamma e polinomial cúbico apresentaram as melhores estimativas para explicar o comportamento do peso dos suínos machos e fêmeas. O modelo logístico apresentou $\mathrm{R}^{2}=0,975$ e AIC (critério de informação de Akaike) $=13,34$ para os machos e $R^{2}=0,9787$ e $A I C=10,16$ para as fêmeas. $O$ modelo gamma apresentou grau de explicação de $R^{2}=0,991$ e $A I C=99,33$ para os machos e $R^{2}=0,9941$ e $A I C=90,602$ para as fêmeas, enquanto que o modelo polinomial cúbico apresentou $\mathrm{R}=0,9995$ e $\mathrm{AIC}=64,2$ para os machos e $\mathrm{R}^{2}=0,9995$ e $\mathrm{AIC}=31,728$ para as fêmeas. Optouse pelo modelo polinomial cúbico para explicar o comportamento do peso simulado dos sú́nos, pois o mesmo apresentou $\mathrm{R}^{2}$ alto e baixo AlC além de menos parâmetros que os outros modelos.

\section{Cooling holding-pen for dairy cows under grazing pasture without shade access}

\section{SUMMARY}

This study aimed to simulate data to the weight of boar and pig sows ( 1 to 154 days old) through the uniform probability distribution on an interval $[a, b]$. Five regression models (logistic, hyperbolic tangent, power, gamma and cubic polynomial) were adjusted to account for the possible weight ratio to tage of pigs in days. The logistic and gamma cubic polynomial models showed the best estimates to account for the weight of the behavior of boar and sow pigs. The logistic model showed $\mathrm{R}^{2}=0.975$ and AIC (Akaike's information criterium)= 13.34 for boars and $R^{2}=0.9787$ and $A I C=10.16$ for sows. The gamma model presented $a$ degree of explanation of $R^{2}=0.991$ and $A I C=99.33$ for boars and $R^{2}=0.9941$ and $A I C=$ 90.602 for sows, while the cubic polynomial model showed $R^{2}=0.9995$ and $A I C=64.2$ for boars and $R^{2}=0.9995$ and $A I C=31.728$ for sows. the cubic polynomial model was chosen to explain the behavior of the simulated weight of the pigs, whose model showed the best fit with fewer parameters than the other models.

\section{INFORMATION}

\section{Cronología del artículo.}

Recibido/Received: 18.08.2016

Aceptado/Accepted: 07.01.2017

On-line: 15.07.2017

Correspondencia a los autores/Contact e-mail:

laura_rocha77@yahoo.com.br

\section{INTRODUÇÃO}

Na suinocultura têm-se desenvolvido estudos sobre curvas de crescimento, sabendo-se que programas de melhoramento genético aliado às pesquisas nutricionais, podem alterar o comportamento biológico do crescimento desses animais, pois modificando-se a estrutura genética de uma população e alterando comportamento alimentar, observam-se mudanças significativas na estrutura de uma raça ou espécie.
Estudar o crescimento ou o desenvolvimento por meio de ajustamento de uma função que descreva todo o período de vida do animal, relacionando peso e idade, tem sido pesquisado por vários autores (Macedo et al., 2014; Morais et al., 2015; Texeira et al., 2012).

A curva descrevendo uma sequência de medidas de determinada característica de alguma espécie ou indivíduo em função do tempo, geralmente peso, altura ou diâmetro, é chamada de curva de crescimento (Fernan- 
des et al., 2012). Em geral, esse tipo de curva apresenta aspecto de crescimento exponencial ou sigmoidal (formato de "S"), que é modelado pela regressão não linear (Fernandes et al., 2012).

As análises de dados de medidas repetidas são de fundamental importância na produção animal, pois incluem as situações em que as unidades experimentais ou indivíduos, de diferentes subpopulações, tratamentos (sexo, raça, entre outros), são analisados ao longo de diversas condições de avaliação tais como tempo, clima e umidade do ar (Freitas, 2005). Vários modelos têm sido propostos para explicar o crescimento biológico dos animais, podendo ser citadas as funções Brody, logística, Gompertz, Von Bertalanffy (Souza et al., 2011; Ó et al., 2012; Silva et al., 2011).

Os estudos sobre curvas de crescimentos em animais vêm sendo explorado por vários autores como relatados em trabalhos de Vargas et al. (2006), que estudaram a modelagem da curva de crescimento em frangos, Morais et al. (2015) verificaram que o modelo quadrático logaritmo é o melhor para explicar o peso em relação a idade de frango, Silva et al. (2011) constataram que os modelos de Brody e Von Bertalanffy foram os mais adequados para explicar o crescimento de bovinos dos grupos genéticos Nelore, 1/2 Anggus + $1 / 2$ Nelore, $1 / 2$ Canchim $+1 / 2$ Nelore e $1 / 2$ Simental $+1 / 2$ Nelore. Já Souza et al. (2010) verificaram que o modelo logístico apresentou o melhor ajuste para descrever o peso dos bovinos da raça InduBrasil.

Na caprinocultura e ovinocultura destacam-se os trabalhos de Malhado et al. (2008) onde verificaram que os modelos de Von Bertalanffy, Gompertz e logístico apresentaram os melhores ajustes para descrever o peso dos caprinos da raça Anglo Nubiana criados na caatinga. Souza et al. (2011) constataram que os modelos de Von Bertalanffy, Brody e Richards foram os mais adequados para explicar o peso dos caprinos da raça Morada Nova criados no estado da Bahia. Texeira et al. (2012) observaram que o modelo de Richards e Brody foram os que apresentaram melhores estimativas para o crescimento do peso de cordeiros. Ó et al. (2012) observaram que os modelos de Gompertz e Von Bertalanffy apresentaram os melhores ajuste para explicar o crescimento dos ovinos da raça Santa Inês. Gbangboche et al. (2008) verificaram que o modelo de Brody foi o mais adequado para explicar o comportamento do peso de ovelhas criadas na África.

Na suinocultura destacam-se os trabalhos de Freitas (2005) onde concluiu que o modelo de Gompertz foi o que melhor explicou o crescimento em suínos, já Oliveira et al. (2007) verificou que o modelo de Von Bertalanfly foi o que melhor explicou o crescimento do peso dos suínos em relação a idade, Luo et al. (2015), Macedo et al. (2014) e Sabbioni et al. (2009) verificaram que o modelo mais adequado ao crescimento de suíno foi o logístico. Vincek et al. (2012) observaram que o modelo de distribuição logística generalizada foi o que melhor explicou a curva de crescimento de suínos. Silva et al. (2013) verificaram que os modelos não lineares de Brody, Gompertz, Von Betarlanffy, Richards e logístico apresentaram estimativas bem próximas para previsão do crescimento de peso em suínos em sistema semi-in- tensivo. Kohn et al. (2007) avaliaram que os modelos não lineares de Richards, logístico e Gompertz foram os que melhor se ajustaram ao peso dos leitões. Schinckel et al. (2003) verificaram que o modelo que melhor explicou a curva de crescimento dos suínos foi a exponencial quadrática.

Não há na literatura um consenso sobre qual modelo melhor explica o comportamento do ganho de peso em suínos relacionados com a idade. Assim, visando ampliar os estudos sobre o assunto, objetivou-se realizar uma análise através de uma série de modelos de regressão para modelar o crescimento do peso de suínos.

\section{MATERIAL E MÉTODOS}

\section{DADOS}

Os dados foram simulados de acordo com valores de peso para suínos, machos e fêmeas, de acordo com os pesos encontrados na literatura, no período de 0 a 154 dias, num intervalo de sete em sete dias. Para cada período de tempo foram simulados cinco observações de uma variável aleatória com distribuição uniforme contínua e sua média calculada.

Seja X uma variável com distribuição de probabilidade uniforme num intervalo $[a, b]$, então definiu-se a função de densidade de probabilidade de X por:

$$
\mathrm{f}(\mathrm{x})=1 /(\mathrm{b}-\mathrm{a}) ; \text { se } a \leq \mathrm{x} \leq b
$$

\section{0; caso contrário}

Para cada período de tempo foram selecionados de forma aleatória cinco observações da distribuição uniforme definida no intervalo $[a, b]$ e depois calculado uma média desses valores utilizando a fórmula abaixo:

$$
\mathrm{x}=a+(b-a) \mathrm{R}
$$

onde, $a$ e $b$ são o menor e o maior valor do peso do suíno para uma determinada idade e R é um valor aleatório entre 0 e 1, assim definimos os valores de pesos simulados. Por exemplo, para o período de tempo de 0 dias e sejam $a=0,9$ e $b=1,5$ e os valores aleatórios de $\mathrm{R}$ iguais a 0,$1 ; 0,25 ; 0,5 ; 0,75$ e 0,95 , respectivamente, então os pesos simulados são:

$$
\begin{aligned}
& x 1=0,9+(1,5-0,9) 0,1=0,96 \\
& x 2=0,9+(1,5-0,9) 0,25=1,05 \\
& x 3=0,9+(1,5-0,9) 0,5=1,2 \\
& x 4=0,9+(1,5-0,9) 0,75=1,35 \\
& x 5=0,9+(1,5-0,9) 0,95=1,47
\end{aligned}
$$

A média dos valores simulados é dado então por 1206 e este valor então é o peso simulado para o tempo 0 dias. Os valores de $a$ e $b$ que geram os intervalos 
de peso (kg) em relação a idade (dias) e sexo dos suínos estão dispostos na tabela I. Na figura 1 encontram-se os valores dos pesos simulados dos suínos de acordo com o sexo e idade, obtidos através da tabela I.

\section{MODELOS DE REGRESSÃO}

Para avaliar a relação entre o ganho de peso dos suínos (machos e fêmeas) e a idade dos mesmos, foram propostos cinco modelos de regressão: logístico, potência, tangente hiperbólica, gamma e polinomial.

\section{Modelo logístico}

Seja X uma variável aleatória com distribuição logística, define-se a função de densidade de probabilidade em [1].

$$
f(x)=\omega \beta \exp (\alpha+\beta X) /(\exp (\alpha)+\exp (\beta X))^{2} \quad \ldots \ldots \ldots \ldots
$$

então, determina-se o modelo logístico por:

$$
Y_{i}=\omega /\left(1+\exp \left(\beta_{0}+\beta_{1} \operatorname{dias}_{i}\right)+\varepsilon_{1} i=1,2,3, \ldots, n\right.
$$

onde, $\mathrm{Y}_{\mathrm{i}}$ é o peso simulado do i-ésimo suíno após o nascimento; dias $_{\mathrm{i}}$ é o i-ésimo dia de avaliação e ci é o i-ésimo erro associado ao peso, em que apresenta

\begin{tabular}{|c|c|c|}
\hline \multirow{2}{*}{ Idade(dias) } & \multicolumn{2}{|c|}{$\begin{array}{l}\text { Intervalos de pesos }(\mathrm{kg}) \\
{[\mathrm{a} ; \mathrm{b}]}\end{array}$} \\
\hline & Machos & Fêmeas \\
\hline 0 & {$[0,9 ; 1,6]$} & {$[0,9 ; 1,5]$} \\
\hline 7 & {$[2,0 ; 3,0]$} & {$[2,5 ; 3,0]$} \\
\hline 14 & {$[3,5 ; 5,0]$} & {$[3,8 ; 5,0]$} \\
\hline 21 & {$[5,1 ; 7,3]$} & {$[5,1 ; 7,0]$} \\
\hline 28 & {$[7,5 ; 9,5]$} & {$[7,5 ; 9,0]$} \\
\hline 35 & {$[9,4 ; 12,0]$} & {$[9,6 ; 11,5]$} \\
\hline 42 & {$[12,1 ; 16,0]$} & {$[12,5 ; 15,5]$} \\
\hline 49 & {$[16,2 ; 20,0]$} & {$[16,8 ; 18,0]$} \\
\hline 56 & {$[20,3 ; 24,0]$} & {$[20,9 ; 22,0]$} \\
\hline 63 & {$[24,2 ; 28,5]$} & {$[24,0 ; 25,5]$} \\
\hline 70 & {$[28,7 ; 32,0]$} & {$[28,5 ; 30,3]$} \\
\hline 77 & {$[32,5 ; 38,0]$} & {$[34,0 ; 35,0]$} \\
\hline 84 & {$[38,2 ; 43,0]$} & {$[39,0 ; 40,2]$} \\
\hline 91 & {$[43,3 ; 49,0]$} & {$[44,2 ; 46,0]$} \\
\hline 98 & {$[49,5 ; 56,0]$} & {$[50,0 ; 52,0]$} \\
\hline 105 & {$[56,3 ; 61,0]$} & {$[57,0 ; 59,0]$} \\
\hline 112 & {$[61,5 ; 69,0]$} & {$[63,8 ; 64,5]$} \\
\hline 119 & {$[69,5 ; 77,0]$} & {$[69,5 ; 71,2]$} \\
\hline 126 & {$[77,3 ; 83,0]$} & {$[76,4 ; 77,8]$} \\
\hline 133 & {$[83,5 ; 91,0]$} & {$[83,0 ; 84,8]$} \\
\hline 140 & {$[91,2 ; 98,0]$} & {$[90,0 ; 91,5]$} \\
\hline 147 & {$[98,7 ; 106,0]$} & {$[96,9 ; 98,4]$} \\
\hline 154 & {$[106,5 ; 115,0]$} & {$[103,8 ; 105,3]$} \\
\hline
\end{tabular}
distribuição normal de média 0 e variância constante

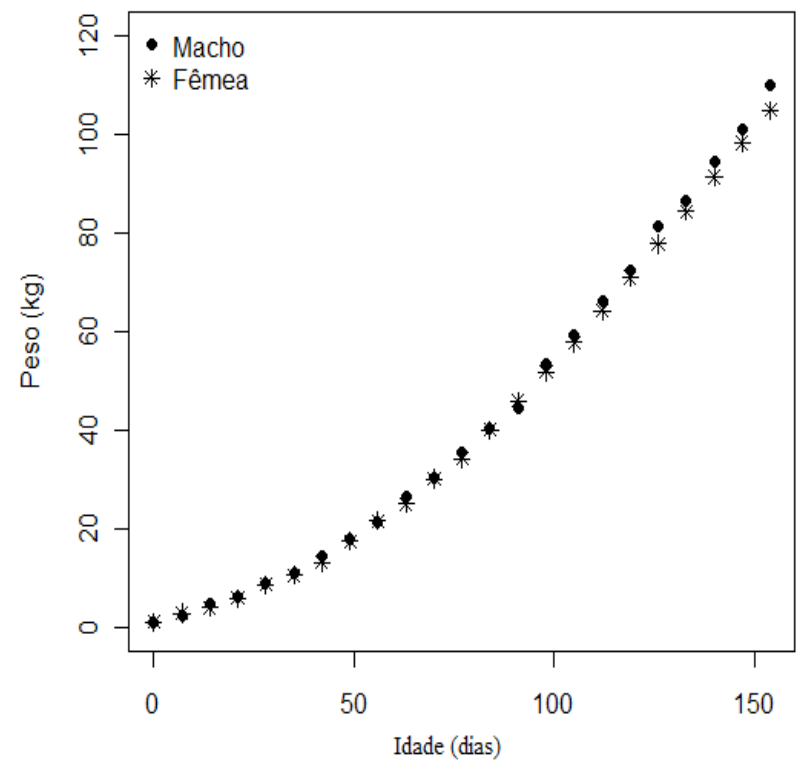

Figura 1. Valores simulados dos pesos dos suínos em relação ao sexo (Simulated values of pig weight in relation to sex).

$\sigma^{2}$. As incógnitas $\omega, \beta_{0}$ e $\beta_{1}$ são os parâmetros associados ao modelo.

\section{MODELO POTÊNCIA}

O modelo de regressão potência é definido em (3).

$$
\mathrm{Y}_{\mathrm{i}}=\beta_{0} \operatorname{dias}_{\mathrm{i}}^{\beta \mathrm{i}} \varepsilon_{\mathrm{i}} \quad \mathrm{i}=1,2,3, \ldots, \mathrm{n}
$$

onde, $\mathrm{Y}_{\mathrm{i}}$ é o peso simulado do i-ésimo suíno após o nascimento; dias ${ }_{i}$ é o i-ésimo dia de avaliação e é o i-ésimo erro associado ao peso, em que apresenta distribuição normal de média 0 e variância constante $\sigma^{2}$. As incógnitas $\beta_{0}$ e $\beta_{1}$ são os parâmetros associados ao modelo.

\section{MODELO TANGENTE HIPERBÓLICA}

Seja X uma variável aleatória com distribuição tangente hiperbólica, então define-se função de densidade de probabilidade em (4).

$$
\mathrm{f}(\mathrm{x})=\beta \gamma \mathrm{X}^{\gamma-1}\left(1-\tanh \left(\beta \mathrm{X}^{\gamma}\right)^{2}\right)
$$

logo, determina-se o modelo tangente hiperbólico por:

$$
Y_{i}=\omega \tanh \left(\beta_{0} \operatorname{dias}_{\mathrm{i}}^{\beta i} \varepsilon_{\mathrm{i}}\right) \quad \mathrm{i}=1,2,3, \ldots, \mathrm{n}
$$

onde, $\mathrm{Y}_{\mathrm{i}}$ é o peso simulado do i-ésimo suíno após o nascimento; dias é o i-ésimo dia de avaliação e é o i-ésimo erro associado ao peso, em que apresenta distribuição normal de média 0 e variância constante $\sigma^{2}$. As incógnitas $\omega, \beta_{0}$ e $\beta_{1}$ são os parâmetros associados ao modelo.

\section{MODELO GAMMA}

Seja X uma variável aleatória com distribuição gamma, define-se a função de densidade de probabilidade em (6).

$$
\mathrm{f}(\mathrm{x})=1 /\left(\Gamma(\alpha) \beta^{\alpha}\right) x^{\alpha-1} \exp (-\mathrm{x} / \beta)
$$



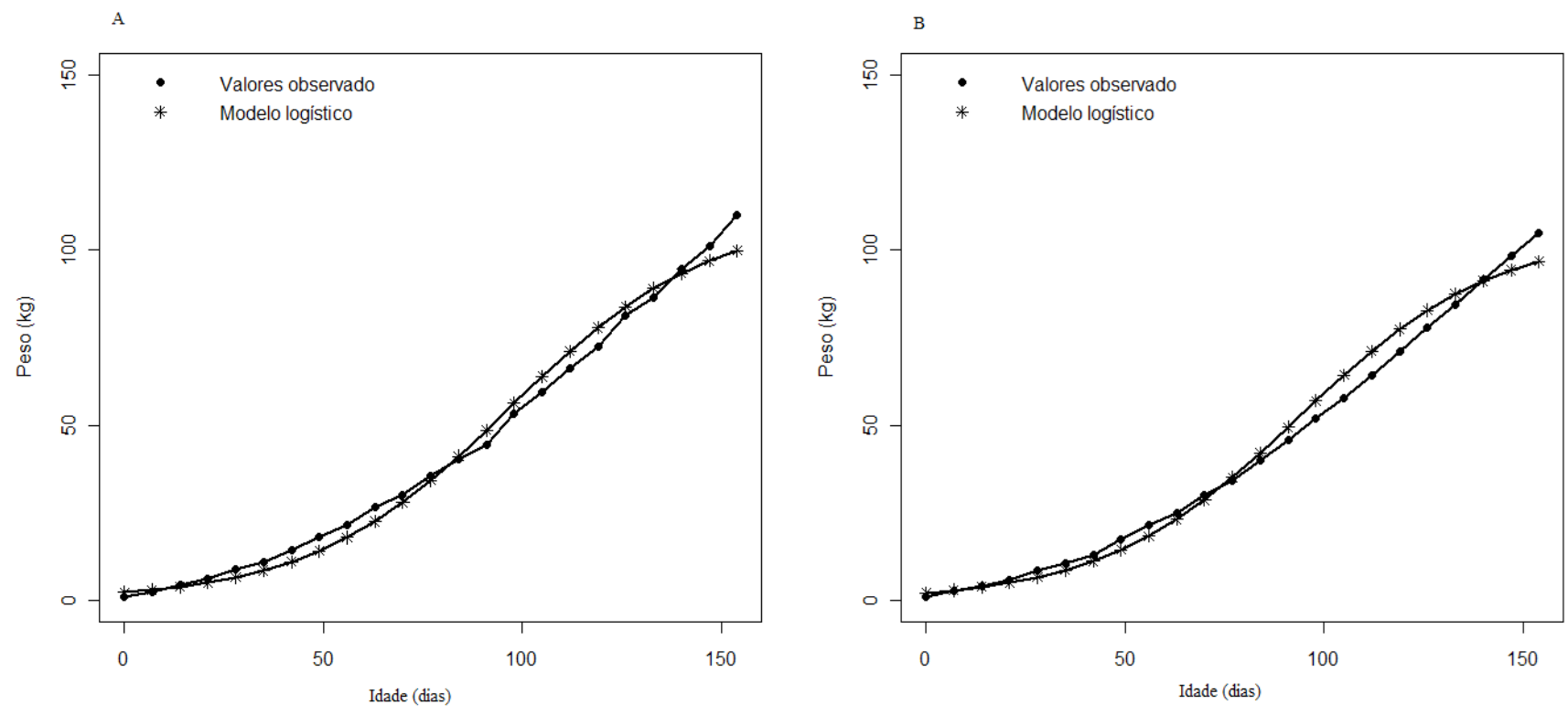

Figura 2. Ajuste do modelo de regressão logístico para peso simulados dos suínos machos (A) e fêmeas (B) (Adjustment of logistic regression model for the simulated weight of male (A) and female (B) pigs).

logo, determina-se o modelo gamma com função de ligação raiz quadrada por:

$$
Y_{i}=\left(\beta_{0}+\beta_{1} d i a_{i}\right)^{2}+\varepsilon_{i}=1,2,3, \ldots, n
$$

onde, $\mathrm{Y}_{\mathrm{i}}$ é o peso simulado do i-ésimo suíno após o

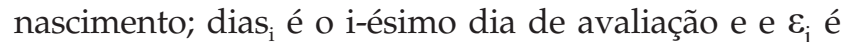
o i-ésimo erro associado ao peso, em que ci apresenta distribuição gamma de parâmetros $\alpha$ e $\beta$. As incógnitas $\beta_{0}$ e $\beta_{1}$ são os parâmetros associados ao modelo.

\section{MODELO POLINOMIAL}

O modelo de regressão polinomial cúbico é definido por [8].

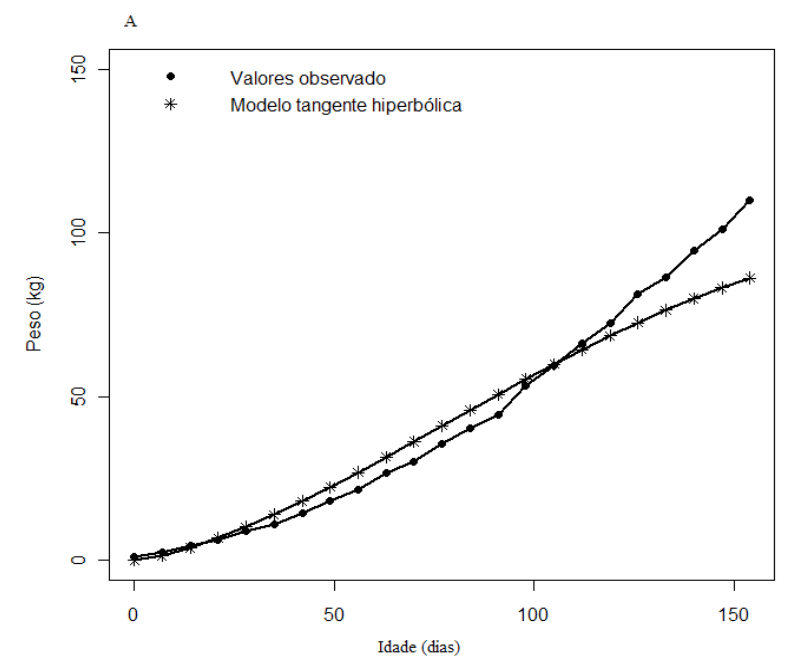

$\mathrm{Y}_{\mathrm{i}}=\beta_{0}+\beta_{1} \mathrm{dia}+\beta_{2}\left(\text { dias }_{\mathrm{i}}\right)^{2}+\beta_{3}\left(\text { dias }_{i}\right)^{3}+\varepsilon_{i} \quad \mathrm{i}=1,2,3, \ldots, 7 \quad[8]$ onde, $\mathrm{Y}_{\mathrm{i}}$ é o peso simulado do i-ésimo suíno após o nascimento; dias $_{\mathrm{i}}$ é o i-ésimo dia de avaliação e $\varepsilon_{\mathrm{i}}$ é o i-ésimo erro associado ao peso, em que $\varepsilon_{1}$ apresenta distribuição normal de média 0 e variância constante $\sigma^{2}$. As incógnitas , $\beta_{0}, \beta_{1}, \beta_{2}$ e, $\beta_{3}$ são os parâmetros associados ao modelo.

\section{CRITÉRIOS DE SELEÇÃO DOS MODELOS}

Os modelos foram avaliados e escolhidos pelos seguintes critérios: maior coeficiente de determinação do modelo $\left(R^{2}\right)$, menores critérios de informação de Akaike (AIC) e soma de quadrados dos resíduos (SQR).

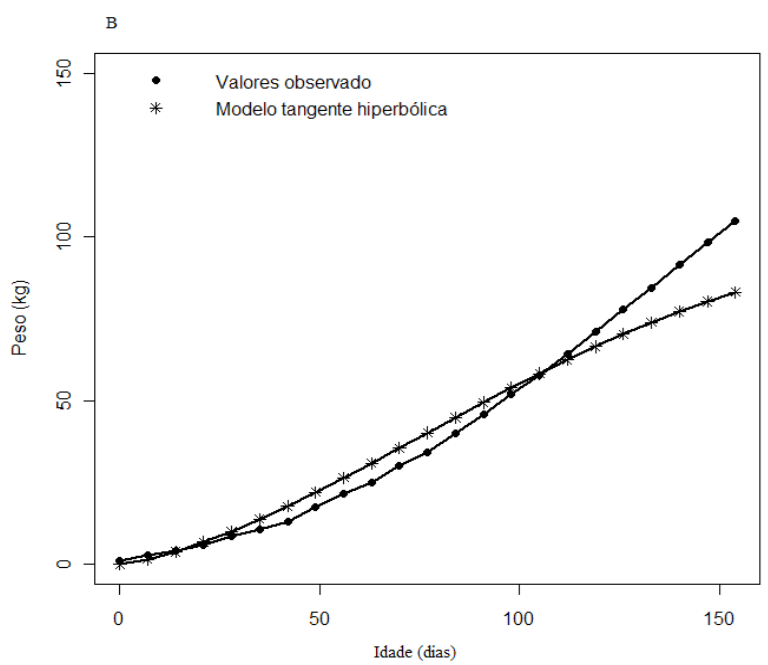

Figura 3. Ajuste do modelo tangente hiperbólica para peso dos suínos machos (A) e fêmeas (B) (Adjustment of the hyperbolic tangent model for the weight of male (A) and female (B) pigs). 
Tabela II. Modelos ajustados e critérios de adequação dos modelos para os suínos machos e fêmeas (Adjusted models and model suitability criteria for male and female pigs)

\begin{tabular}{|c|c|c|c|c|}
\hline \multirow{2}{*}{ Modelos } & \multirow{2}{*}{ Equação ajustada } & \multicolumn{3}{|c|}{ Critérios de adequação do modelo } \\
\hline & & $\mathrm{R}^{2}$ & AIC & SQR \\
\hline \multicolumn{5}{|l|}{ Machos } \\
\hline Logístico & $=109,93 / 1+\exp \left(3,874-0,041\right.$ dias $\left._{\mathrm{i}}\right)$ & 0,975 & 13,34 & 305,73 \\
\hline Potência & $=0,123 \operatorname{dias}_{i}^{1,32}$ & 0,9837 & 19,25 & 740,09 \\
\hline T. hiperbólica & $=109,93 \tanh \left(0,00079 \operatorname{dias}_{\mathrm{i}}^{1,429}\right)$ & 0,9618 & 3,38 & 34,813 \\
\hline Gamma & $=\left(1,056+0,064 \operatorname{dias}_{\mathrm{i}}\right)^{2}$ & 0,991 & 99,33 & 239,667 \\
\hline P. cúbico & $=1,598+0,104 \operatorname{dias}_{\mathrm{i}}+0,00492 \operatorname{dias}_{\mathrm{i}}^{2}-0,0000066 \operatorname{dias}_{\mathrm{i}}^{3}$ & 0,9995 & 64,2 & 14,34 \\
\hline \multicolumn{5}{|l|}{ Fêmeas } \\
\hline Logístico & $=104,96 / 1+\exp \left(3,848-0,041\right.$ dias $\left._{\mathrm{i}}\right)$ & 0,9787 & 10,16 & 331,108 \\
\hline Potência & $=0,13 \operatorname{dias}_{i}^{1,3}$ & 0,9747 & $-9,95$ & 775,223 \\
\hline T. hiperbólica & $=104,96 \tanh \left(0,00085 \operatorname{dias}_{\mathrm{i}}^{1,418}\right)$ & 0,9497 & 9,061 & 33,278 \\
\hline Gamma & $=\left(1,105+0,062 \operatorname{dias}_{\mathrm{i}}\right)^{2}$ & 0,9941 & 90,602 & 145,232 \\
\hline P. cúbico & $=1,759+0,071 \operatorname{dias}_{i}+0,0055 \operatorname{dias}_{i}^{2}-0,00001 \operatorname{dias}_{i}^{3}$ & 0,9999 & 31,728 & 8,887 \\
\hline
\end{tabular}

AIC: Critério de informação de Akaike; SQR: soma de quadrados dos resíduos.

Seja o i-ésimo valor do peso dos suínos após ajuste do modelo, então defini-se a soma dos quadrados dos resíduos para este estudo pela seguinte expressão:

$$
\mathrm{SQR}=\sum_{i=1}^{n}\left(Y_{i}-\widehat{Y}_{i}\right)^{2}
$$

O coeficiente de determinação do modelo $\left(R^{2}\right)$ é expresso pela razão entre a soma de quadrado do modelo (SQM) e a soma de quadrados total (SQT), ou seja,

$$
\mathrm{R}^{2}=\frac{\mathrm{SQM}}{\mathrm{SQT}}=1-\mathrm{SQR} / \mathrm{SQT}=1-\frac{\sum_{i=1}^{n}\left(Y_{i}-\hat{Y}_{2}\right)^{2}}{\sum_{i=1}^{n}\left(Y_{i}-Y_{l}\right)^{2}}
$$

O critério de informação de Akaike (AIC) definido por Akaike (1974) é dado por:

$$
\mathrm{AIC}=-2 \ln \mathrm{L}(\mathrm{x} \backslash \hat{\theta})+2(\mathrm{p})
$$

onde, $L(x \backslash \theta)$ é a função de máxima verossimilhança, definida como sendo o produtório da função de densidade e p é quantidade de parâmetros do modelo.

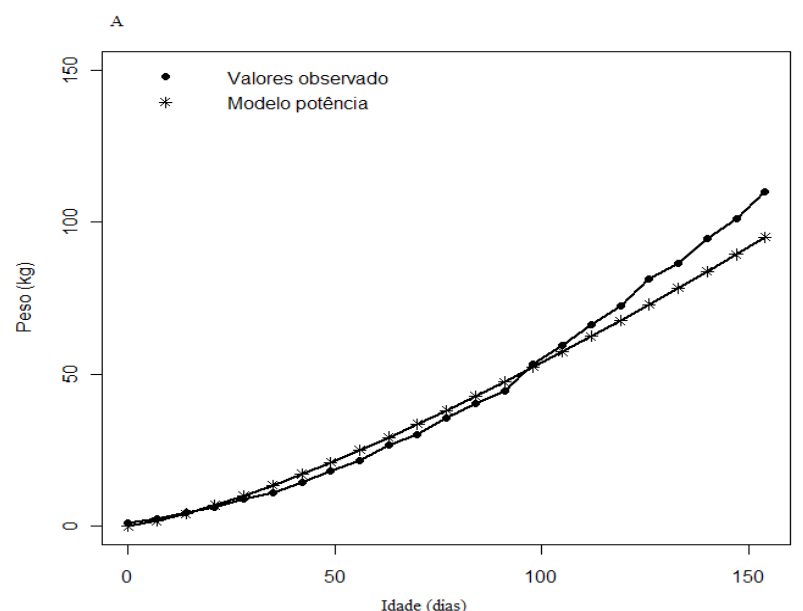

\section{RESULTADOS}

Verificou-se que o modelo logístico apresentou estimativas satisfatórias dos pesos simulados dos suínos machos e fêmeas para todo o período de avaliação (figura 2). Tanto para os suínos machos como para as fêmeas o modelo logístico apresentou elevado poder de explicação $\left(R^{2}=0,975\right.$ e 0,9787$)$ e baixo $\mathrm{AIC}=13,34$ e 10,16, respectivamente. O modelo ainda apresentou $\mathrm{SQR}=305,73$ (machos) e 331,108 (fêmeas) (tabela II). Apresentando comportamento similar aos pesos simulados dos suínos fêmeas até o $98^{\circ}$ dia, entre o $98^{\circ}$ e $133^{\circ}$ dia, demonstrando ajustes superiores aos valores simulados, e após o $133^{\circ}$ dia o modelo apresentou estimativas inferiores às observadas.

O modelo tangente hiperbólica apresentou estimativas próximas aos pesos simulados dos suínos machos e fêmeas até o $126^{\circ}$ dia (figura 3A e 3B). Após o $126^{\circ}$ dia o modelo passou a subestimar os valores observados. $\mathrm{O}$ modelo apresentou $\mathrm{R}^{2}=0,9618, \mathrm{AIC}=3,38$ e SQR $=34,813$

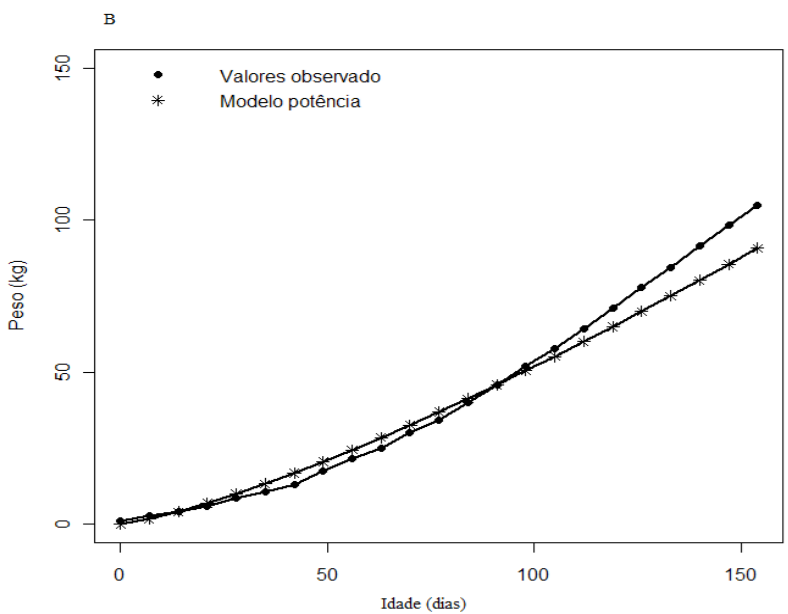

Figura 4. Ajuste do modelo potência para peso dos suínos machos (A) e fêmeas (B) (Adjustment of the power model for the weight of male (A) and female (B) pigs). 
para os machos e $\mathrm{R}^{2}=0,9497, \mathrm{AIC}=9,061$ e $\mathrm{SQR}=33,278$ para as fêmeas (tabela II). Assim, como o modelo tangente hiperbólica, o modelo potência apresentou ajuste dos pesos dos suínos machos e fêmeas semelhantes aos simulados. Após o $126^{\circ}$ dia o modelo passou a subestimar os valores observados (figura 4A e 4B), apresentando um $\mathrm{R}^{2}=0,9837, \mathrm{AIC}=19,25$ e $\mathrm{SQR}=740,09$ para os suínos machos e $\mathrm{R}^{2}=0,9747, \mathrm{AIC}=-9,95$ e $\mathrm{SQR}=775,223$ para os suínos fêmeas (tabela II). Os modelos (hiperbólico e potência) explicaram o peso simulado dos suínos machos e fêmeas apresentando bons ajustes até o $126^{\circ}$ dia. Notou-se que o modelo gamma apresentou bons ajustes para explicar o peso simulado dos suínos machos e fêmeas em todo o período avaliado até o $133^{\circ}$ dia (figura 5A e 5B). O modelo apresentou um alto poder de explicação tanto para os suínos machos $\left(R^{2}=0,991\right)$ como para as fêmeas $\left(R^{2}=0,9941\right)$, AIC de 99,33 para os machos e de 90,602 para as fêmeas e SQR de 239667 para os machos e de 145232 para fêmeas (tabela II).

Observou-se que o modelo polinomial cúbico apresentou bons ajustes tanto para os pesos dos suínos ma- chos quanto para as fêmeas, tais ajustes são similares aos pesos simulados dos suínos machos e fêmeas em todo o período avaliado (figura 6A e 6B). Com excelente poder de explicação $\left(R^{2}=0,9995\right.$ para os machos; $R^{2}=0,9999$ para as fêmeas), $\mathrm{AIC}=64,2$ para os machos e $\mathrm{AIC}=31,728$ para as fêmeas e SQR de 14,34 para os machos e 8,887 para fêmeas (tabela II).

\section{DISCUSSÃO}

De acordo com os critérios de avaliação dos modelos ( $\mathrm{R}^{2}, \mathrm{AIC}$ e SQR), os que melhor se ajustaram ao comportamento do peso simulado foram o logístico, gamma e polinomial cúbico, tanto para o peso simulado dos suínos machos como para as fêmeas. Com os coeficientes de determinação para os modelos acima de $94 \%$, demonstrando que os dados simulados no trabalho foram estimados adequadamente. Sendo, os modelos gamma e polinomial cúbico superiores a 0,99. Lopes et al. (2011) estudando a estimativa da curva de crescimentos em bovinos encontraram coeficientes de
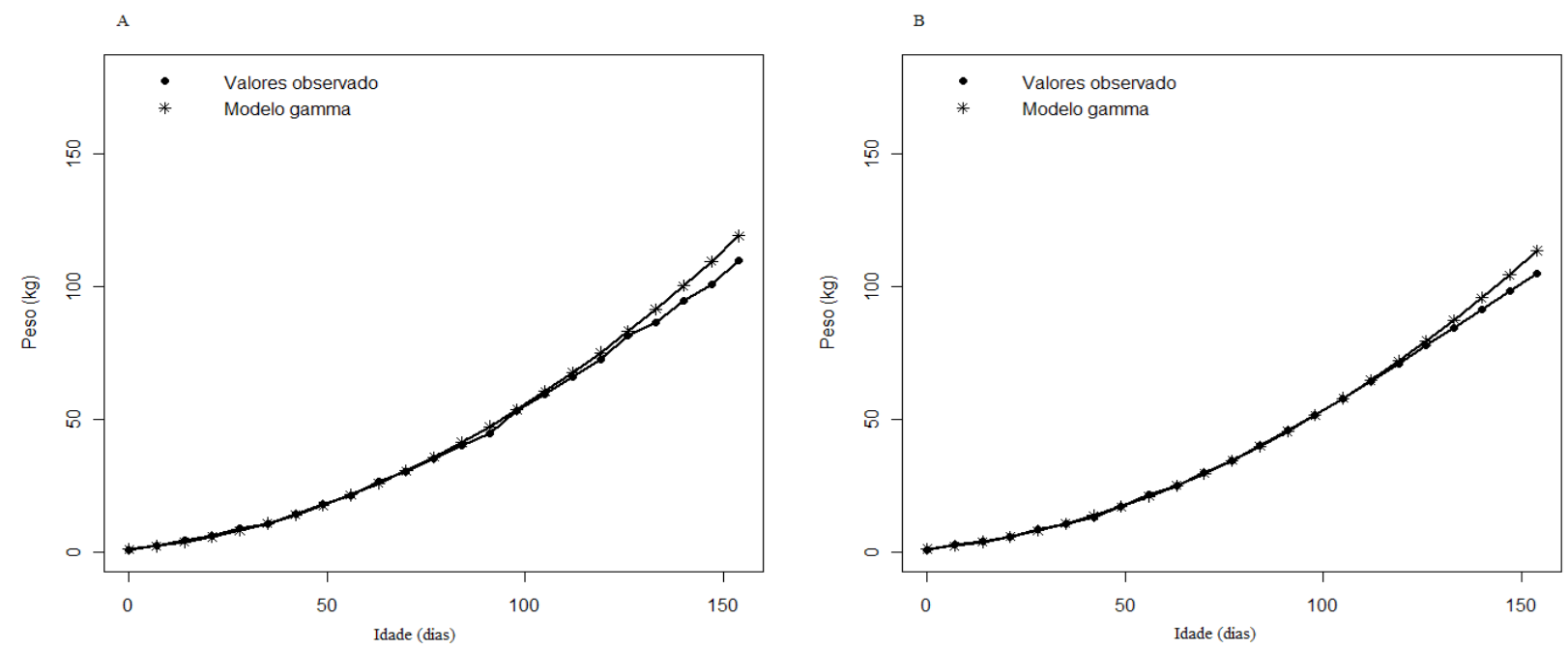

Figura 5. Ajuste do modelo de regressão gamma para peso dos suínos machos (A) e fêmea (B) (Adjustment of the gamma regression model for weight of male $(A)$ and female $(B))$.
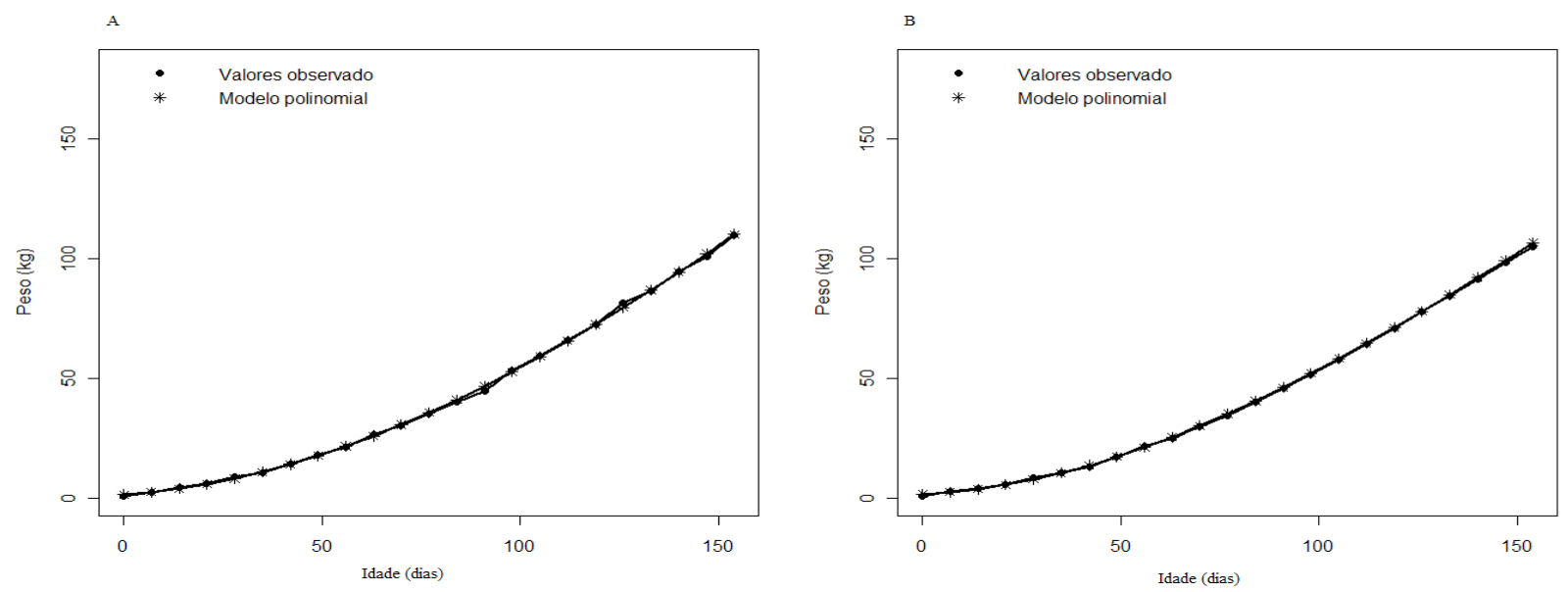

Figura 6. Ajuste do modelo de regressão polinomial cúbico para peso dos suínos machos (A) e fêmeas (B) (Adjustment of the cubic polynomial regression model for the weight of male (A) and female (B) pigs). 
determinação semelhantes para o modelo logístico, apresentando taxa de crescimento inferior aos demais modelos a partir dos quatro meses. Já Luo et al. (2015) verificaram que os modelos logístico, Von Bertalanffy e Gompertz apresentaram estimativas superiores a 99\%, fornecendo precisões ótimas para o crescimento do peso dos suínos.

As avaliações com os cinco modelos propostos permitiram que se realizasse inferência sobre o estágio de desenvolvimento do suíno. Estudos vêm demonstrando que o uso de modelos aplicados ao desenvolvimento animal permitiu resultados satisfatórios quanto ao desenvolvimento do animal. A utilização do desenvolvimento dos tecidos ósseo, muscular e adiposo em suínos podem ser aplicados com boa acurácia para estudar curvas de crescimentos em suínos (Dutra Jr. et al., 2001; Vincek et al., 2012), havendo a necessidade de avaliar diferentes modelos e métodos estatísticos para que possam descrever com precisão o desenvolvimento do animal.

Vários são os parâmetros para a escolha do melhor modelo que explique o desenvolvimento do animal. Muianga et al. (2013) para a determinação do modelo que explicasse melhor a curva de crescimento em suínos, escolheram os modelos que apresentaram os melhores valores para $\mathrm{R}_{\text {ajd }}^{2}$ AIC e critério Bayesiano. Veloso et al. (2016) em estudos de modelos para descrever a curva de crescimento de frangos de corte, descreveram a qualidade dos ajustes dos modelos pelo critério de qualidade do coeficiente de determinação dos ajustes, critério de AIC e bayesiano, quadrado médio do erro e pelo índice de assintótico. Dentre os modelos estudados, o modelo polinomial cúbico foi definido como o que melhor explicou o comportamento dos pesos simulados dos suínos machos e fêmeas, tendo como critério de escolha o $\mathrm{R}^{2}$, AIC e SQR, permitindo predizer o peso de acordo com a idade do suíno, podendo ser utilizado em futuras avaliações de crescimento de suínos de corte, onde a curva estimada no modelo polinomial cúbico é igual para os dois sexos, o que não acontece nos demais modelos avaliados.

\section{CONCLUSÃO}

Diversos fatores podem influenciar na qualidade do ajuste do modelo, como peso, idade, sexo, o manejo utilizado, a própria raça, o presente trabalho demonstrou que os critérios de escolha do modelo podem ser utilizados para futuros estudos de curvas de crescimento em suínos, auxiliando em programas de seleção.

\section{BIBLIOGRAFIA}

Akaike, H. 1974. A new look at the statistical model identification. IEEE Trans Automat Contr, 19: 716-723.

Coutinho, C.C. 2014. Curvas de crescimento de características de carcaça obtidas por ultrassonografia em bovinos nelore selecionados para peso pós desmama. Universidade Estadual Paulista. Faculdade de Ciências Agronômicas. Botucatu. 60 pp

Dutra Jr., W.M.; Ferreira, S.; Donzele, J.L.; Euclydes, R.F.; Tarouco, J.U. e Cardoso, L.L. 2001, Predição de curvas de crescimento de tecidos de fêmeas suínas por intermédio da função alométrica estendida. Rev Bras Zootecn, 30: 1007-1014.

Fernandes, T.L.; Pereira, A.A. e Muniz, J.A. 2012. Comparação de modelos não-lineares no estudo das curvas de crescimento do caranguejo de água doce. Revista da Estatística da Universidade Federal de Ouro Preto, 2: 2375-2387.

Freitas, A.R.D.E. 2005. Curvas de crescimento na produção animal. Rev Bras Zootecn, 34: 786-795.

Freitas, A.R.D.E. 2007. Estimativas de curva de crescimento na produção animal. Embrapa Pecuária Sudeste. Documentos 68.

Gbangboche, A.B.; Glele-Kakai, R.; Salifou, S.; Albuquerque, L.G. and Leroy, P.L. 2008. Comparison of non-linear growth models to describe the growth curve in West African Dwarf sheep. Animal, 2: 1003-1012.

Kohn, F.; Sharifi, A.R. and Simianer, H. 2007. Modeling the growth of the goettingen mini pig. J Anim Sci, 85: 84-92.

Luo, J.; Lei, H.; Shen, L.; Yang, R.; Pu, Q.; Zhu, K.; Li, M.; Tang, G.; Li, X.; Zhang, S. and Zhu, L. 2015. Estimation of growth curves and suitable slaughter weight of the Liangshan pig. Asian Australas J Anim Sci, 28: 1252-1258.

Macedo, L.R.; Silva, F.F.; Cirillo, M.A; Nascimento, M.D.M.P.; Paes, S.E.F.G.; Lopes, O.S.; Santos, J.A. e Azevedo, C.F. 2014. Modelagem hierárquica bayesiana na avaliação de curvas de crescimento de suínos genotipados para gene halotano. Rev Ciênc Rural, 44: 1853-1859.

Malhado, C.H.M.; Carneiro, P.L.S.; Cruz, J.F.; Oliveira, D.F.; Azevedo, D.M.M.R. e Sarmento, J.L.R. 2008. Curvas de crescimento para caprinos da raça anglo-nubiana criados na caatinga: rebanho de elite e comercial. Rev Bras Saúde Prod Anim, 9: 662-671.

Morais, J.; Ferreira, P.B.; Jacone, I.M.T.D.; Mello, R.; Brenda, F.C. e Rorato, P.R.N. 2015. Curva de crescimento de diferentes linhagens de frango de corte caipira. Rev Ciênc Rural, 45: 1872-1878.

Muianga, C.A.; Nascimento, M.D.A.S.;Fernandes, T.J. e Muniz, J.A. 2013. Ajuste dos modelos logístico e Gompertz aos dados de crescimento de suínos. Anais do XII Encontro Mineiro de Estatística. MGEST. Uberlândia.

Ó, A.L.; Neto, A.U.R.; Santos, G.V.; Sarmento, J.L.R.; Biagiotti, D.; Souza, J.E.R. 2012. Curva de crescimento de ovinos Santa Inês no vale do Gurgueial. Rev Bras Saúde Prod Anim, 13: 912-922.

Oliveira, L.A.J.V.B. e Bassanezi, R.C. 2007, Modelo de Von Bertalanffygeneralizado aplicado ao crescimento de suínos de corte. Biomatemática, 17: 101-109.

Sabbioni, A.; Beretti, V.; Manini, R.; Cervi, C. and Superchi, P. 2009. Application of different growth models to Nero di Parma pigs. Italian J Anim Sci, 8: 537-539.

Schinkel, A.P.; Ferrell, J.; Einsten, M.E.; Pearce, S.A. and Boyd, R.D. 2003. Analysis of pig growth from birth to sixty days of age. Swine Research Report. Purdue University. 10 pp.

Silva, F.L.; Alencar, M.M.; Freitas, A.R.; Packer, I.U. e Mourão, G.B. 2011. Curvas de crescimento em vacas de corte de diferentes tipos biológicos. Pesq Agropec Bras, 46: 262-271.

Silva, F.F.; Resende, M.D.V.; Rocha, G.S.; Duarte, D.A.S.; Lopes, P.S.; Brustolini, O.J.B.; Thus, S.; Viana, J.M.S. and Guimarães, S.E.F. 2013. Genomic growth curves of an outbred pig population. Genet Mol Biol, 36: 520-527.

Souza, L.A.; Caires, D.N.; Carneiro, P.L.S.; Malhado, C.H.M. e Filho, R.M. 2010. Curvas de crescimento em bovinos da raça Indubrasil criados no estado de Sergipe. Rev Ciênc Agron, 41: 671-676.

Souza, L.A.; Carneiro, P.L.S.; Malhado, C.H.; Paiva, S.R.; Caires, D.N. e Barreto, D.L.F. 2011 . Curvas de crescimento em ovinos da raça Morada Nova criados no estado da Bahia. Rev Bras Zootecn, 40: 1700- 1705. Texeira, M.C.; Villarroel, A.B.; Pereira, E.S.;Oliveira, S.M.P.;Albuquerque, I.A. e Mizubuti, I.Y. 2012. Curva de crescimento de cordeiros de três sistemas de produção na região nordeste do Brasil. Rev Ciênc Agron, 33: 2011-2018

Vargas, G.D.; Dionello, N.L.; Brum, P.A.R.; Rutz, F. e Fialho, F.B. 2006. Modelagem de crescimento e do desenvolvimento de frangos de corte: validação. Ciênc Rural, 36: 1664-1669.

Veloso, R.C.; Winkelstroter, L.K.; Silva, M.T.P.; Pires, A.V.; Torres Filho, R.A.; Pinheiro, S.R.F.; Costa, L.S. e Amaral, J.M. 2016, Seleção e classificação multivariada de modelos não lineares para frangos de corte. Arq Bras Med Vet Zootec, 68: 191-200.

Vincek, D.; Sabo, K.; Kusec, G.; Kralik, G.; Durkin, I. and Scitovski, R. 2012. Modeling of pig growth by $S$-function least absolute deviation approach for parameter estimation. Archiv Tierzucht, 55: 364-374. 